IDEAS IN ECRLGGY AND EVRLUTION 5: 57-62, 2012

doi:10.4033/iee.2012.5.13.c

(C) 2012 The Author. (C) Ideas in Ecology and Evolution 2012

Received 8 June 2012; Accepted 25 September 2012

Commentary

\title{
The shifting states of resilience: Easier to define than to measure
}

\author{
Beatrix E. Beisner \\ Beatrix E. Beisner (beisner.beatrix@uqam.ca),Department of Biological Sciences, University of Quebec at \\ Montreal, C.P. 888, Succ. Centre-Ville, Montreal, QC, H3C 3P8, Canada
}

\begin{abstract}
In this response to Myers-Smith et al (2012), I take a historical perspective on the different types and components of resilience so as to provide a comprehensive conceptual framework that could be applied to alleviate the confused use of the term in the literature as identified. At one level, the apparently divergent definitions arise as a result of an evolution of ecological interest from a local stability perspective to one of global stability where alternative stable states are possible. It is my view that the confusion about definitions of resilience in the literature quantified by Myers-Smith et al. originates from a lack of clarity about this fact, as well as a lack of understanding of a heavily term-laden field where alternative stable states are concerned. I commend MyersSmith et al. for clearly pointing out the problem regarding the use of the term resilience by ecologists. I hope that this commentary will further help ecologists remedy the problem through more consistent usage, by enabling a more complete comprehension of the development of ideas about resilience and using a simple case study to review a common conceptual framework.
\end{abstract}

Key Words: perturbation, local stability, global stability, alternative stable states, hysteresis

\section{Introduction}

Myers-Smith et al. (2012) clearly demonstrate that there has been sloppy and often confused use of the term resilience in the literature. I believe that this problem stems from three sources: (i) a definition that has evolved through time as ecologists have expanded their view from single to multiple state systems, (ii) different perspectives in the study of alternative states that change how resilience might be defined and (iii) a difficulty in actually quantitatively measuring resilience in a multiple states context. I commend Myers-Smith et al. for their literature survey and for exposing the problem in a quantitative way. It should serve as a wake-up call to the community to revisit the theory of alternative stable states and to clarify meanings when it comes to this complicated term. But I think some more clarity on the perspectives is required if this is to happen. I will attempt to add some clarity to the discussion by taking a historical perspective and reviewing the components of resilience. Ultimately, despite the confused literature and conclusions of Myers-Smith et al. (2012), I believe that there is a unifying paradigm for resilience.

\section{History of an ecological idea}

Exclusive of the early work by Holling (1973), much of ecology that addressed resilience until around 2000 revolved around testing stability in single state systems where only local stability applied. Thus, the definitions of Pimm (1991) and Carpenter et al. (1992) in MyersSmith et al.'s (2012) Table 1 were most often used: resilience was most simply the return time to a previous equilibrium after a pulse perturbation. Ecological systems were considered to be rather well-behaved, that is linear and without complex interactions that might lead to "surprises" in the dynamics. This was largely a period during which the focus was on local stability: small perturbations from equilibrium.

However during the 1990's, field ecologists began accumulating more and more surprises, and observing important shifts in their study systems in response to perturbations, be they continuous (press) or discrete (pulse) perturbations. Some classic examples came from coral reefs (Knowlton 1992, Hughes 1994), others from 
plant-animal-parasite interactions in the Serengeti (Dublin et al. 1990), yet others from shallow lakes (Scheffer 1993). These studies recognized the connection to earlier theory on alternative stable states (Holling 1973, Noy-Meir 1975, May 1977) and this led to a revival of interest. What became apparent is that nonlinearities are more common than expected and that where these occur, one must start to consider global, and not just local, stability. Within such a framework, the measurement of resilience as the time required to return to an original state becomes less meaningful because of a very important feature of alternative states: hysteresis. Hysteresis can be thought of as non-linearity in return times of systems to their previous states (for a full discussion see Beisner et al. (2008)). Because of hysteresis, a complex system may never return to its former state, or if it does, it will do so at a different critical tipping point (measured in units of the variable being perturbed) going backward than it did going forward with the original perturbation. Taking a global stability perspective led ecologists dealing with such complex systems to adopt more widely, and to further refine, Holling's (1973) definition of resilience (e.g. Gunderson 2000, Carpenter et al. 2001, Walker et al. 2004 in Table 1 of Myers-Smith et al. 2012 are all versions of this). Recognizing this conceptual shift early on, Peterson et al. (1998) identified the local stability definition as engineering resilience and the more complicated one referring to a global stability context as ecological resilience. Specifically, ecological resilience is "the measure of the amount of change or disruption that is required to transform a system from being maintained by one set of mutually reinforcing processes and structures to a different set of processes and structures" (Peterson et al. 1998:10). This definition referred to the fact that when considering stability of a state in a multiple stable state context, one is considering aspects of global stability. There are different basins of attraction and it becomes the characteristics of these basins of attraction that are the focus. Essentially, if a system "escapes" its basin of attraction following a perturbation, then it is considered less resilient than a system that remains within its basin given the same perturbation. However, I believe the problems and confusion (as identified by Myers-Smith et al. 2012) occur when ecologists try to measure the "amount of change or disruption" required, as I will discuss next.

\section{More than one way to leave your state}

I believe that a large part of the confusion about the term resilience arises because there are actually two very different ways of moving a complex system from one state to another, leading to uncertainty as to how resilience should be measured quantitatively. While Scheffer et al. (2001; Figure 2) and Walker et al. (2004) do describe these different paths between states, I believe this fact is not clear to most ecologists who do not actively work with the theoretical constructs of alternative stable states ecology and may thus tend to use the term resilience more loosely than they should. We (Beisner et al. 2003a) also began to address the issue by identifying two different perspectives on alternative stable states, but I would now like to expand on that viewpoint by more specifically considering how resilience itself is assessed.

Let's consider a well-known case of alternative stable states and one that is close to my heart (Beisner et al. 2003b): the propensity for algae (A) to bloom in lakes with high phosphorus (P) loads. I will start with the diagram in Figure 1a which is a common way of representing complex systems where there are alternative states (if you are interested in how this is generated from first principles, which will only muddy the main point here, there are many published examples including Scheffer et al. 2001, Beisner et al. 2003b, Beisner 2012). In such phase space plots for systems with alternative states, there is generally a slowly changing variable along the $\mathrm{x}$-axis ( $\mathrm{P}$ loading from the landscape to our lake in Figure 1a is considered to occur over many years) and a variable that responds quickly to this first one on the $y$-axis (A, which can respond on the order of days, to a change in $\mathrm{P}$ in Figure 1a). From the solid part of the S-curve on the graph, it is easy to see that for intermediate levels of $\mathrm{P}$, both a low and a high A stable state are possible; that is, there are alternative stable states in A present at these values of our slow variable $\mathrm{P}$. The upper solid line represents the high A state and the lower solid line the low A state. The dotted line between these represents the edge of the basins of attraction of each state. These S-curves can be transformed into the commonly presented heuristic schematic in Figure 1b.

Now we can ask: how do we get our system to move from a low A state to a high A state when we are in the region of intermediate $P$ (the dot in Figure 1a)? Understanding this will allow us to better grasp what is meant by resilience and to identify sources of confusion around the issue. As mentioned, there are two ways to do this. One is to modify the fast responding population/ community variable A directly. For our lake ecosystem this could be done in a number of ways as long as the end result is a lot of algal biomass (A) being added to the lake in short order. For now, let's assume that a biofuels facility growing algae next to the lake had a large spill that ended up in the lake one day. If the spilled algal biomass was enough to move A from the lower state, up to and just beyond (at least) the unstable state (dashed part of the S-curve; Figure 1a), then we would see a shift to the upper A state. In this case, resilience is defined as the direct distance between the stable state and its basin boundary ( $\mathrm{L}$ arrow in 
(a)

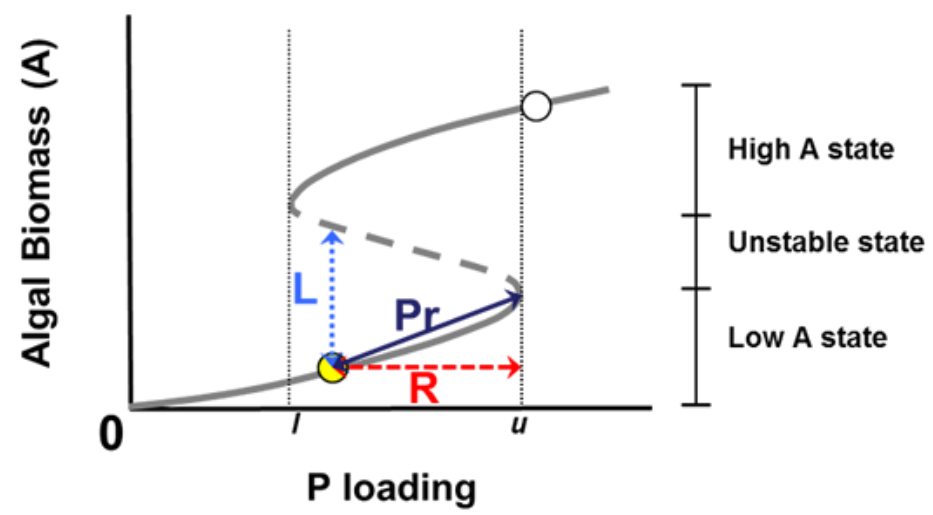

(b)

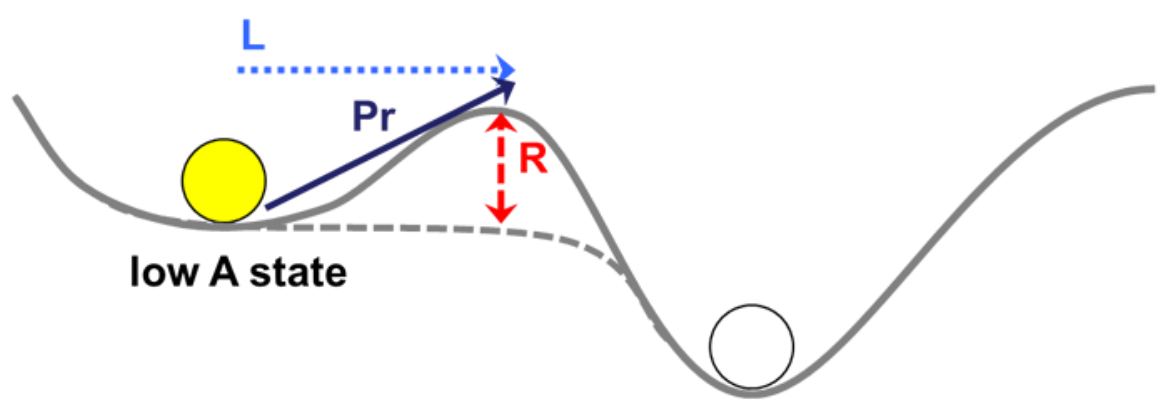

high A state

Figure 1. Diagrams showing the possible system states for the algal-phosphorus system in terms of (a) phase space (joint values) where the solid yellow point shows the starting state for discussion of resilience that follows. The dotted lines indicate the value of $\mathrm{P}$ for which there is a critical (tipping) point from the low A to the high A stable state $(u)$ and the value of $\mathrm{P}$ for which there is a critical point from the high A back to the low A state $(l)$. In (b) is shown the corresponding and commonly used heuristic ball-in-cup diagram where the ball represents the state of the algal biomass (A) and the cup or landscape represents the value of $\mathrm{P}$ loading. In both panels the various components of ecological resilience discussed in the text are shown: latitude (L), resistance $(\mathrm{R})$ and precariousness $(\mathrm{Pr})$.

Figure 1). Equivalently in Figure 1b, the distance to the edge of the cup is what defines resilience of a particular state. In Beisner et al. (2003a) we referred to this type of change in the variables of the study system as the "community perspective." In Walker et al. (2004), they called this the "latitude" component of resilience.

The second way to move the A-P system between states is by adding enough $\mathrm{P}$ to the lake from the landscape over time such that the $\mathrm{P}$ load eventually exceeds the upper critical point (shown as a dotted vertical line $u$ in Figure 1a). By slowly adding $\mathrm{P}$ (moving right along the x-axis; Figure 1a), we overcome the "resistance" resilience of the lower A state by eroding the size of its basin of attraction and move the entire system to the right along the lower stable A line.
Adding P amounts to a change in the "landscape" of the ball-in-cup diagram as shown in Figure 1b (dashed landscape line) and this is what we referred to as the "ecosystem perspective" in Beisner et al. (2003a). In this diagram, resilience changes as the landscape changes, and can be thought of most simply as the amount of $\mathrm{P}$ that we would need to add to our lake to reduce the height of the hill separating the basins to nothing (equivalently, the depth of the basin of attraction; $\mathrm{R}$ arrow in Figure 1b). In Walker et al. (2004), this is what they refer to as the "resistance" component of resilience.

The issue gets more complicated, however, when we realize that while we are modifying $\mathrm{P}$ (moving to the right along the $\mathrm{R}$ arrow in Figure 1a), we are also 
reducing the amount of $\mathrm{A}$ (size of the corresponding $\mathrm{L}$ arrow) needed to shift states. For this reason, the resilience of the system under study might be better represented by some combination of the vectors describing the height of the hill and the width of the basin ( $\mathrm{P}$ arrow in Figure 1). Walker et al. (2004) call this "precariousness": essentially a measure of how close the system is to a threshold in the combination of ways in which it can move between states.

Finally I would like to return to the first manner in which I discussed how a state shift might occur in our A-P system to demonstrate a final aspect of resilience that was also described by Walker et al. (2004): panarchy. We were concerned with how we might move between states by directly adding lots of algal biomass (A). I gave an extreme example of how this might be done; extreme because it is unlikely that one could grow enough algae to cause any lake to shift between states (a small pond perhaps). Instead, a more common way this would be done is through a trophic cascade: reducing herbivory by zooplankton on A, by adding a fourth trophic level of piscivorous fish to a lake with only planktivorous fish present. An issue arises because the addition of fish is a dynamic considered to be external to our focal system defined (i.e. we are not considering fish dynamics in our model directly), because fish also have their own dynamics and at particular timescales that may not match those of our focal A-P system. If a small number of piscivorous fish invaded our lake without our knowledge and if their population gradually grew over time, this would amount to the presence of another slow variable (in addition to $\mathrm{P}$ ) with ultimate effects on A. Because the fish dynamics probably operate at different spatial and temporal scales than does the $\mathrm{P}$ addition (eutrophication) upon which we are focussed, fish could affect our system through A directly (latitude) and/or P (resistance). Once the fish population reached some critical threshold of its own, it could lead to an unexpected state shift (algal bloom) for a given level of P. The potential interference or inter-action of this unobserved/unmeasured dynamical variable (fish) with the focal study system (A-P) is an aspect of what Walker et al. (2004) refer to as "panarchy". Ultimately, the resilience of a focal system may depend on such unmeasured components within which a study system is embedded (Carpenter et al. 2001).

Ultimately, I believe that the source of confusion about resilience identified by Myers-Smith et al. (2012) arises because, unless one has followed the development of ideas in the alternative stable states/resilience literature, one is quickly lost by what appears to be a lot of jargon. However, this jargon refers to some clearly different pathways to how a state shift might occur: (i) directly to the focal-dependent fast variable (latitude), (ii) through a slowly-changing focal variable (resistance) or (iii) through the combined effects of these (precariousness). The only way to circumvent confused usage of resilience in the literature is by ensuring that ecologists are more generally aware of the conceptual framework for global stability and the associated critical dynamics. At minimum, a proper referencing in the literature to ecological and engineering resilience would be a start in alleviating the issue raised by Myers-Smith et al (2012). But also, authors will need to clearly discuss and measure the slow and fast variables in their systems to ensure that they can identify which aspect of resilience it is they are quantifying.

\section{Frames of reference for resilience}

Recognizing the historical and conceptual contexts in which different definitions of resilience have been used allows us to address the concern raised by Myers-Smith et al. (2012) regarding the frame of reference for comparison of resilience and the different metrics. The comparative nature of resilience is independent of how the ecological system under study is assessed or measured because it is should always be relative to the previously unperturbed state in the "fast" variable of interest. Whether a study measures population densities, ecosystem flows or community structure will likely affect the degree (quantity, units) of resilience, but it does not change the definition of the term. That is, if the situation is one of a pulse perturbation and of local stability, engineering resilience will be defined with respect to the original, unperturbed state, irrespective of what actual properties of the ecosystem are being measured. It is with respect to historic conditions that values are compared. Similarly, where a space-for-time substitution is used, one could imagine that resilience could be evaluated with respect to other similar, but unperturbed study systems. To say that ecological resilience is greater means that there is a larger basin of attraction (width, depth and/or both) for the state of interest, making it more likely for that ecological system to remain within it. Resilience can thus be measured on different ecosystem components, but one needs to be clear about the state of which variable is of interest so that responses can be compared to those in similar ecosystems from other regions or in other experiments. For our A-P case study, the latitude (L) component of resilience would be measured in terms of A (algal biomass), the resistance $(\mathrm{R})$ component in terms of $\mathrm{P}(\mathrm{P}$ input rate) and the precariousness ( $\mathrm{Pr}$ ) component in terms of some combined value of these (note: I must admit to being unsure as to how this aspect would be measured, perhaps as L*P?). Admittedly this does mean that it may be difficult to directly compare absolute resilience between very different types of ecosystems such as a forest vs. a plankton community but that is the case regardless, for most other ecological metrics because of important differences in life histories, 
behaviours and habitat features. Relative measures of resilience (i.e. resilience as a proportion of the actual state) could be applied however, to allow comparison in meta-analyses.

\section{Quantifying resilience}

As noted by Myers-Smith et al. (2012), few studies have attempted to quantify resilience: in large part this is because it is often difficult to measure, and in addition to not being always well understood conceptually. For engineering, resilience quantification is more easily done, especially in an experimental context because, when systems are more well-behaved (linear, fast dynamics) and easy to experimentally manipulate or assess, one can measure return time relatively easily. To do so empirically for ecological resilience is much more difficult. In order to quantify resilience in this case, one needs to estimate the size of the basin of attraction for the state of interest; and if considering changes in the slow variable, at which point the hill between basins is fully (or almost) eroded.

Basins of attraction, while relatively easy to discuss heuristically and to define theoretically (as in our Figure 1), are notoriously hard to define empirically. First, alternative stable states imply non-linear interactions of some type. Be it the response of a fish population to harvesting which, when greater than some critical point reduces the population to zero, or a lake that "flips" suddenly into an algal-dominated turbid state after years of nutrient addition, the critical points at which these events occur are the ones that define the boundaries of the system state. As we have seen, distance of the current state to the boundary (horizontally and/or vertically) represents aspects of its ecological resilience. Thus to measure ecological resilience, we need to know the values of the slow variable between which the fast variable shows alternative stable states. Returning to our example, we need to know the lowest P level at which a high A state first becomes a possibility ( $u$ in Figure 1a) and we need know at which upper P level the low A state disappears completely ( $l$ in Figure 1a). Knowing these values, as well as the upper and lower stable state relationships between $\mathrm{P}$ and $\mathrm{A}$ (solid line curves in Figure 1a), we can draw the useful S-curve and estimate resilience of the low A state (for example) for any joint $\mathrm{P}$ and $\mathrm{A}$ values in our lake. It seems simple in theory, but the sad truth is that we have such little knowledge of real ecological systems that it is very difficult to define these boundaries of basins of attraction, except for the most well-studied systems (some examples in Folke et al. 2004). Most empirical studies operate in the absence of a theoretical model which is parameterized for the study system (for obvious reasons), making it difficult to know where to look within gradients for nonlinearities and potential critical points. Another problem arises with measurements: what might in reality be a non-linear relationship will too often be interpreted as linear given the noise in field ecological data and our tendency to assume parsimony when fitting statistical models. These problems mean that we have difficulty even defining whether a state is alternative or not, never mind what the boundaries of the basin of attraction might be for the original one so as to estimate resilience. As a result, we are more often likely to discuss aspects of populations, communities or ecosystems that might help "build" resilience by keeping the system in as deep and wide basin of attraction as possible.

\section{Conclusions}

Overall, I agree with Myers-Smith et al.'s (2012) assessment results: resilience is ill-defined in too many studies and this is unacceptable. It is clear that there are not only different types of resilience but different components of it, as I have tried to review and clarify here. Solutions have been proposed, initially by Peterson et al. (1998) and more recently by Walker et al. (2004) and together with an understanding of the theory of alternative stable states (Beisner et al. 2003a), resilience in its various components is actually quite simply defined both heuristically and theoretically. And, a unifying framework can be considered (Fig. 1). The problem lies in estimating resilience empirically in natural ecological systems. However, I believe that ecology as a field needs to acknowledge and embrace the challenge of quantitative estimates of ecological resilience. A more quantitative understanding of global stability would allow ecologists to better predict and, when necessary, to prevent unwelcome surprises that are characteristic of so many complex ecological systems. To do so, we need to ensure that ecologists have a good handle on the theoretical basics of complex dynamical systems and this starts with a clear use of terminology that we can apply to our noisy, complex natural systems. It is my hope that this commentary will help in some small way to improve this situation identified by MyersSmith et al (2012).

\section{References}

Beisner, B.E., D. Haydon and K.L. Cuddington. 2003a. Alternative stable states in ecology. Frontiers in Ecology and the Environment 1:376-382. CrossRef

Beisner, B.E, Dent L. and S.R. Carpenter. 2003b. Variability of lakes on the landscape: roles of phosphorus, food webs, and dissolved organic carbon. Ecology 84:1563-1575. CrossRef

Beisner, B.E., Haydon, D. and K.L. Cuddington. 2008. Ecological Models: Hysteresis. Pages 1930-1935 in Jorgensen, S.E., and B.D. Fath, editors. 
Encyclopedia of Ecology, Volume 3, 1st Edition. Elsevier B.V., Oxford. CrossRef

Beisner, B.E. 2012. Alternative Stable States. Nature Education Knowledge, in press.

Carpenter, S.R., Walker, B., Anderies, J.M. and N. Abel. 2001. From metaphor to measurement: resilience of what to what? Ecosystems 4:765-781. CrossRef

Carpenter, S.R., Kraft, C.E., Wright, R., He, X., Soranno, P.A. and J.R. Hodgson. 1992. Resilience and resistance of a lake phosphorus cycle before and after food web manipulation. American Naturalist 140:781-798. CrossRef

Dublin, H.T., Sinclair, A. R. and J. McGlade. 1990. Elephants and fire as causes of multiple stable states in the Serengeti-Mara woodlands. Journal of Animal Ecology 59:1147-1164. CrossRef

Gunderson, L. Ecological resilience-in theory and application. Annual Review of Ecology and Systematics 31:425-439. $\underline{\text { CrossRef }}$

Holling, C.S. 1973. Resilience and stability of ecological systems. Annual Review of Ecology and Systematics 4:1-24. $\underline{\text { CrossRef }}$

Hughes, T. P. 1994. Catastrophes, phase shifts, and large-scale degradation of a Caribbean coral reef. Science 265:1547-1551. CrossRef

Knowlton, N. 1992. Thresholds and multiple stable states in coral reef community dynamics. American Zoologist 32:674-682.
May, R.M. 1977. Thresholds and breakpoints in ecosystems with a multiplicity of states. Nature 269:471-477. CrossRef

Myers-Smith, I.H., Trefry, S.A., and V.J. Swarbrick. 2012. Resilience: Easy to use but hard to define. Ideas in Ecology and Evolution 5: 44-53. CrossRef

Noy-Meir, I. 1975. Stability of grazing systems: An application of predator-prey graphs. Journal of Ecology 63:459-481. CrossRef

Peterson G., Allen C.R. and C.S. Holling. 1998. Ecological resilience, biodiversity and scale. Ecosystems 1:6-18. CrossRef

Pimm, S.L. 1991. The Balance of Nature? Ecological Issues in the Conservation of Species and Communities. University of Chicago Press, Chicago, USA.

Scheffer, M., Hosper, S. H., Meijer, M. L. and B. Moss. 1993 Alternative equilibria in shallow lakes. Trends in Ecology and Evolution 8: 275-279. CrossRef

Scheffer M., Carpenter S.R., Foley J.A., Folke C. and B. Walker. 2001. Catastrophic shifts in ecosystems. Nature 413:591-596. CrossRef

Walker, B., Holling, C.S., Carpenter, S., and A. Kinzig. 2004. Resilience, adaptability and transformability in social-ecological systems. Ecology and Society $9: 5$ 